\title{
First Aid Guidelines for Allergic Reaction, Seizure and Diabetic Emergency: Educational Approach and Adaptation
}

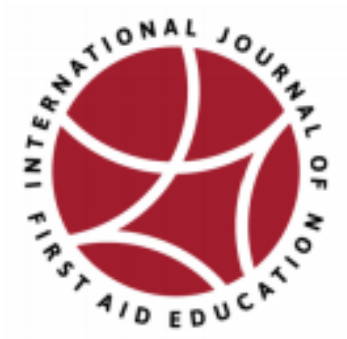

Željko Malić $^{1}$, Panagiotis Ioannidis ${ }^{2}$

${ }^{1}$ Slovenian Red Cross, ${ }^{2}$ International Committee of the Red Cross

The updated First Aid, Resuscitation and Education Guidelines of the International Federation of the Red Cross and Red Crescent Societies (IFRC, 2020) reflect the evidence regarding specific first aid clinical actions and pair it with the evidence regarding first aid education. This approach was designed so that users of the Guidelines are easily able to apply them through adaptation to relevant learner contexts, contextualization to different levels of resources and medical care, and local implementation strategies.

For medical conditions such as allergic reaction, seizure, and diabetic emergency, the Chain of Survival Behaviors represents the domains for education to systematically address the needs of people in a position to aid another or themselves, based on clinical guidelines. Through the development of education strategies in each domain, it supports a system of high quality and accessible first aid.

For the three medical topics reviewed in this paper, the consistent advice is to recognize the medical 'event' as an emergency, call EMS, and to give first aid. For Allergic Reaction, educators are encouraged to create scenarios highlighting the importance of assessment of the situation, finding the cause of the reaction (allergen), and helping the person urgently. Actions such as assisting with medication and emphasizing the correct usage are also suggested. For Seizure, early recognition is key by knowing the signs and symptoms of seizure and encourages bystanders to begin first aid by protecting the person from harm (such as from hitting their head). For Diabetic Emergencies, again, awareness and recognition are crucial precursors to taking the action.

Whilst the Guidelines make a clear effort to identify specific educational content to support learners to recognize and manage the frightening symptoms which can present with these three topics, there are clear limitations and gaps with the evidence available.

There is a need to design research observing the success of implementation progress at a local level. Furthermore, there is a need to design research observing the impact on learners in first aid education curricula, with special attention to those that are less likely to report helping behavior.

The International Federation of the Red Cross and Red Crescent Societies highlighted the importance and connection of first aid education in its recent publication of the 2020 International First Aid, Resuscitation, \& Education Guidelines (IFRC, 2020). First aid education helps people engage in more helping behaviors. Furthermore, it helps populations to live more safely, be more resilient, and provide care when needed. For medical conditions such as allergic reaction, seizure, and diabetic emergency, the Chain of Survival Behaviors (IFRC, 2016) represents the domains for education to systematically address the needs of people in a position to aid another or themselves, based on clinical guidelines. Through the development of education strategies in each domain, it 
supports a system of high quality and accessible first aid.

The International Federation of the Red Cross and Red Crescent Societies updated its 2016 Guidelines (IFRC, 2020) to reflect the evidence regarding specific first aid clinical actions and uniquely paired it with the evidence regarding first aid education. This approach was designed so that users of the Guidelines are easily able to apply them through adaptation to relevant learner contexts, contextualization to different levels of resources and medical care, and local implementation strategies.

The development of each new guideline and the review of the 2016 version included a new focus on the domains of the Chain of Survival Behaviors (IFRC, 2016) as well as a fresh look through an educational lens across different contexts and the provision of education considerations for each topic. This shift in emphasis occurred in response to calls from developers of curricula and first aid educators from across the Movement (including National Societies and the International Committee of the Red Cross - ICRC). The process to include these elements was rigorous and consistent and is explained in full in the Guidelines (IFRC, 2020). Topics for inclusion were identified in 2018 using surveys and expert opinion of actors within the Movement. Individuals from 43 countries with clinical and/or educational expertise participated in teams to develop search criteria from existing published literature and grey literature for each research question.

Following a clinical review of evidence, each topic was then considered independently by at least two educational reviewers (including one from a lower resource setting and one from a higher resource setting) who both reviewed existing literature to inform the educational approach to a first aid intervention for that topic. They also drew on their own experience and expertise. Together they then synthesized the insight available and contributed educational aspects to the Chain of Survival Behaviors for that topic. They also provided Education considerations to support the application of the topic in an educational setting. Any conflicts were resolved by an independent Guidelines Steering Committee (GSC) member. Worksheets that described the outcomes of each review were completed. These were presented for peer review to the GSC.

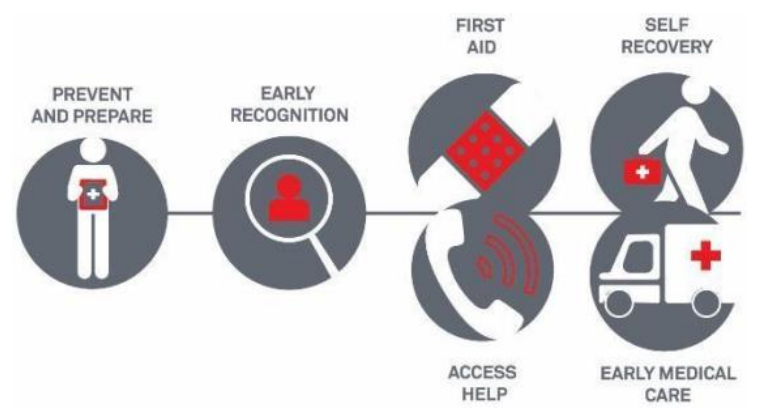

This paper reviews three of the medical conditions covered in the Guidelines. It explores how educational evidence and expert opinion has been added to clinical insight to enable learners to recognize these conditions and act appropriately for maximum harm reduction.

Scientific Foundation

- For the topic Allergic reaction, the data show that allergies are relatively common in the general population (WHO, 2012). Allergic reaction presents on the skin as hives, itching and swelling, or in the airways as sneezing and snuffles. Sometimes it is accompanied by gastrointestinal symptoms. Furthermore, some people can be severely allergic, 
which can lead to a life-threatening anaphylactic reaction (IFRC, 2020). In a life-threatening situation delayed injection of epinephrine is associated with higher hospitalization and mortality rates. The Guidelines present the need for first aid education to focus on the 'Recognition' of allergic reaction and anaphylaxis in order to be able to act effectively.

- Data shows that 442 million adults have diabetes worldwide (WHO, 2016). This prevalence is important when considering the topic of Diabetic Emergency. We also need to take into consideration children diagnosed with diabetes. In the "Prevent and Prepare" domain of the Chain of Survival Behaviors, the Guidelines suggest teaching people with diabetes and first aid providers to alert family, friends, and others about their condition. Furthermore, they advise how to respond in situations of emergency and where to find the diabetic person's medication or blood testing kits. A high blood sugar level (hyperglycemia) may evolve gradually, and a low blood sugar level (hypoglycemia) can evolve suddenly and become a life-threatening situation (IFRC, 2020).

- In the general population Seizures are a medical condition described as a dramatic event. Because of a lack of recognition of what is happening, frequently, bystanders do not know or feel confident to give first aid to someone who is having a seizure. A seizure is usually not harmful and passes after a short time, but they can occasionally cause the person to injure themselves further (IFRC, 2020). Again, for this topic, education on the 'Recognition' phase of the Chain of
Survival Behaviors is an important focus for learners.

The Guidelines introduce each of these conditions with a key action to be emphasized to learners, a brief explanation of the condition, how common it is and the main population likely to be at risk or affected. The Guidelines that follow are rated by the level of scientific evidence that exists for them. Good practice points are provided where evidence was missing but expert opinion was available based on experience and non-systematic review sources of evidence.

The Chain of Survival Behaviors is used by the Guideline authors as a tool to emphasize how first aid education does not start and end with a first aid action. The domains of Prevent and Prepare; Early Recognition; First Aid and/ or Access help; and Early Medical Care/ Self-recovery, are fundamental considerations for education depending on the context and learner needs. Each clinical topic covered by the Guidelines includes insight based on evidence and expert opinion on how best to frame an educational intervention using these domains and identifying where educators might best position that learning.

\section{Educational approach}

For the three medical topics reviewed in this paper, the consistent advice is to recognize the medical 'event' as an emergency, call EMS and to give first aid (IFRC, 2020).

Key actions are juxtaposed with an educational approach, for example:

\section{Allergic reaction}

- The Chain of Survival Behaviors highlights three main areas of focus: 
prevent and prepare, recognize and first aid steps. The aim is to describe "generic educational aspects" that is, raising awareness, recognizing signs and symptoms of a severe allergic reaction, removing the allergen, helping the person to take their medication and access EMS as soon as possible (IFRC, 2020).

Developing decision-making scenarios based on signs and symptoms can help learners to recognize the significance of the situation and develop their own critical thinking (IFRC, 2020).

Learners are advised to stop further contact with the allergen, and scenarios can be pre-designed in situations involving allergens known to be present in a local community (e.g., bees, dust, nuts, etc.) (IFRC, 2020).

Educators are encouraged to create scenarios for an allergic reaction highlighting the importance of assessment of the situation, finding the cause of the reaction (allergen) and helping the person urgently. Actions such as assisting with medication and emphasizing the correct usage (IFRC, 2020) are also suggested.

\section{Seizure}

- The Guidelines emphasize the key action to protect a person from harm (IFRC, 2020).

- Again, the three main areas of focus for learners are prevent and prepare, recognize and first aid steps. For this topic, early recognition by the person may be key to preventing more harm. Awareness can be raised in learners by helping them to recognize signs and symptoms of seizure and encouraging bystanders to give first aid by protecting the person from harm (such as from hitting their head). Additionally, self-awareness of the person suffering from seizure can be helpful if they can recognize the "aura" (a feeling of approaching seizure). From an educational aspect the learner needs to understand in which circumstances he/she needs to access EMS and how to monitor a person who is having a seizure (IFRC, 2020).

\section{Diabetic emergency}

- "When a diabetic emergency occurs, give the person something sweet to eat or drink to raise their blood sugar level" is the key action in Guidelines in the case of low blood sugar (IFRC, 2020).

- Although this is a very practical action to take in response to the situation, from a generic educational perspective, awareness and recognition of a diabetic emergency are crucial precursors to taking the action. The Guidelines emphasize the educational need to raise alertness of the family and others where the person with diabetes is living. Preparedness and early recognition are essential so first aid can be given (IFRC, 2020).

- The Guidelines highlight the need to act swiftly, but caution that we can only give the person something to eat or drink if they are responsive and able to swallow (IFRC, 2020). 
These three first aid topics lend themselves well to activities where the learner can explore and discuss behavior in an emergency situation. This can effectively increase the learner's propensity to act (Oliver et al., 2014). Implementing Guideline recommendations into first aid education on topics of this kind can have a positive impact beyond the intervention itself. For instance, the medical condition of seizure has negative connotations amongst some populations where people suffering from seizure are stigmatized (IFRC, 2020). Increased knowledge about the condition and understanding how to care for a person having a seizure is proven to have a positive impact (O’Hara, 2007).

Van de Velde et al (2011) propose that consideration of ethnic and religious backgrounds, cultural perceptions and understanding of stigma should be addressed and discussed as part of an effective mechanism for implementing the Guidelines, followed by measures or criteria to support their practical application.

\section{Adaptations}

The Guidelines posit that program designers should be mindful of local legislation, regulations, and the availability of relevant solutions in their own contexts. For example, with anaphylaxis the first aid guideline is the administration of adrenaline/ epinephrine, but in many countries, it may not be administered by non-medical professionals. Moreover, in some contexts, adrenaline/ epinephrine autoinjectors are available and could be an important consideration in the discussion about the action of first aiders. These factors can have an effect on what should be taught.
Educators preparing and planning for a course also need to be aware of different contexts and resources (such as distance from EMS or hospitals or supplies available to learners). In high-risk environments, or remote areas where access to EMS can be challenging, clear actions to take need to be taught and understood by lay responders for each of these medical emergencies. In these cases, focus should be education on preparedness and prevention, such as ensuring people carry adequate supplies of medication with them if they are going to a remote area, and learning what to do if you are alone (IFRC, 2020).

In addition, the Guidelines emphasize the need for practising emergency scenarios which take into account the local conditions and help the learner to think through how best to respond effectively.

The approach of the subject from an educational perspective should be adapted to reflect the local understanding and challenges. Definition of the educational approach should come after understanding of the local dimensions of the problem, usually through community participation by organizing focus group discussions and qualitative studies in the local population. Whilst it may be the case that many people who have diabetes are already aware of how to care for themselves, the possibility that a person might encounter an undiagnosed type 1 diabetic or a complication of any other case should be considered when learning about this topic. Where a condition might present as "altered mental status" (such as a diabetic emergency) education needs to raise awareness of the condition and the serious consequences of not responding quickly and effectively. This might be especially important in communities 
where there is prejudice against people who suffer disproportionately from diabetes (Simmons, 2002). Education should include efforts to combat the stigma of the people living with conditions such as diabetes or epilepsy.

Learner considerations are highlighted in the Guidelines (IFRC, 2020). A good educational strategy is needed to consider how we can differently prepare people (IFRC, 2020; Belgian Red Cross-Flanders, 2018). Facilitation tips and tools are described in the Guidelines for these topics (IFRC, 2020).

\section{Discussion}

For the three conditions covered in this paper, considerations beyond the immediate first aid intervention are evidenced as important. These include legal aspects in relation to medication administration, and attitudinal considerations of the conditions. Therefore, to fully achieve the goal of implementing these Guidelines into the local environments, advocacy should be taken into account. Advocacy is defined as "giving public support" to an idea or in our example a course of action (Oxford University Press, 2021).

Whilst the Guidelines make a clear effort to identify specific educational content to support learners to recognize and manage the frightening symptoms which can present with these three topics, there are clear limitations and gaps with the evidence available. For all three topics, the educators could add health promotion messages in the form of promoting the use and raising awareness of the existence of special health tags (medical identification tags), which are somehow common in the Western world but quite rare in the rest of the world (Alraee, 2020). Known life-threatening allergies to any allergen (food, drug, or sting), could therefore be indicated to facilitate and guide the first aid provider.

There is considerable challenge when teaching how to deal with a diabetic emergency due to the largely unspecific manifestations which can severely confuse any lay person, especially in the absence of any hints (diabetic tag) or access to diagnostic tools. Therefore, familiarization with the tools (e.g. epinephrine injector, glucometer) on a theoretical or even practical level could also be considered in places where their existence is common or expected.

In some instances, cultural differences in approach to first aid may create barriers to adopting guidelines or teaching them. Educators need to adapt approaches to fostering essential elements of guidelines. In particular all three topics are riddled with myths and misconceptions that vary greatly across the world. For example, in places where Voodoo is prevalent, epileptic seizures might be attributed to spiritual possession. Similarly, in some areas in Africa seizures are surrounded by "an atmosphere of fear, shame and mysticism" related to a both sacred and demonic condition, under the term corpus sacer (Jilek-Aall, 1999). Causes for diabetes and anaphylaxis are also subject to a great deal of discussion with regards to their actual risk factors (i.e. how possible it is to go into anaphylaxis from a vaccine) (CDC, 2021 as opposed to Nokleby, 2006, also Fletcher 2002).

We would argue that especially for these particular three topics, it is important that educators go beyond communicating the clinical management to touch on the more farreaching objectives of first aid training. 
According to the IFRC First Aid Policy, part of these objectives is to promote respect for diversity and human dignity and reduce intolerance, discrimination, and social exclusion. For epilepsy, for example, there is evidence that misconceptions persist even amongst more developed countries, manifesting with socially exclusionary attitudes, ignorance about treatment, and stigmatizing overgeneralizations (Herrmann, 2016). Equally laden are the topics of both diabetes (Rai, 2009) and anaphylaxis (Anagnostou, 2019), showing that the general population still lags behind the knowledge that is up to par with the international guidelines.

\section{Conclusion}

Evidence-based education strategies can provide a high-quality first aid education. The 2020 Guidelines do not replace first aid manuals, but rather they serve as foundation resources for first aid program designers. There is a need to design research observing the effectiveness of implementation progress at a local level. Furthermore, there is a need to design research observing the impact on learners in first aid education curricula, with special attention to those that are less likely to report helping behavior.

\section{Acknowledgements}

We wish to thank the reviewers, writers and editors that were involved in the development of the 2020 Guidelines.

\section{Conflict of Interests}

The authors were content contributors to the International First Aid, Resuscitation, and Education Guidelines 2020.

\section{Corresponding Author}

Mr Željko Malić email: zeljko.malic@,rks.si

\section{References}

Alraee, S., Albalawi, A., Alshowmer, S., Kinani, S., Alharthi, R., Jawdat, D., \& Chachar. Y. (2020). Exploring the awareness of medical identification tag and its uses in emergency situations in Saudi Arabia. SJEMed, 1(2), 58-62. https://doi.org/10.24911/SJEMed/72-1588628720

Anagnostou, K., \& Turner, P.J. (2019). Myths, facts and controversies in the diagnosis and management of anaphylaxis. Arch Dis Child, 104(1), 83-90. https://doi.org/10.1136/archdischild-2018-314867

Bandstra, N., Camfield, C., \& Camfield, P. (2008). Stigma of Epilepsy. Canadian Journal of Neurological Sciences / Journal Canadien Des Sciences Neurologiques, 35(4), 436-440. https://doi.org/10.1017/S0317167100009082

Belgian Red Cross-Flanders.. (2018). Teaching first aid to children in Africa. Belgian Red Cross-Flanders. https://www.globalfirstaidcentre.org/resource/teaching-frist-aid-to-children-in-africa/

CDC (2021). Allergic Reactions Including Anaphylaxis After Receipt of the First Dose of Pfizer-BioNTech COVID19 Vaccine-United States, December 14-23, 2020. MMWR. Morbidity and Mortality Weekly Report, 70. https://doi.org/10.15585/mmwr.mm7002e1

Fletcher, B., Gulanick, M., \& Lamendola, C. Risk factors for type 2 diabetes mellitus. J Cardiovasc Nurs, 16(2), 17-23. https://doi.org/10.1097/00005082-200201000-00003

Herrmann LK., Welter, E., Berg, A.T., Perzynski, A.T., Van Doren, J.R., \& Sajatovic, M. (2016). Epilepsy misconceptions and stigma reduction: current status in Western countries. Epilepsy \& Behavior, 60, 165-173. https://doi.org/10.1016/j.yebeh.2016.04.003 
Review

International Federation of Red Cross Red Crescent Societies. (2016). International First Aid and Resuscitation Guidelines 2016.

International Federation of Red Cross Red Crescent Societies. (2020). International First Aid, Resuscitation, and Education Guidelines 2020. https://www.globalfirstaidcentre.org/resource/international-first-aid-resuscitation-andeducation-guidelines-2020-2/

Jilek-Aall, L. (1999). Morbus sacer in Africa: some religious aspects of epilepsy in traditional cultures. Epilepsia. 40(3):382-6. https://doi.org/1111/j.1528-1157.1999.tb00723.x

O'Hara, K.A. (2007). First Aid for Seizures: The Importance of Education and Appropriate Response. Journal of Child Neurology, 22(5_suppl), 30S-37S. https:// doi.org/10.1177/0883073807303066

Oliver, E., Cooper, J., \& McKinney, D. (2014). Can first aid training encourage individuals' propensity to act in an emergency situation? A pilot study. Emergency Medicine Journal, 31, 518-520. http://dx.doi.org/10.1136/emermed-2012-202191.

Oxford University Press. (2021). Oxford English Dictionary. Open access: https://www.oxfordlearnersdictionaries.com/definition/english/advocacy?q=advocacy.

Nokleby, H. Vaccination and anaphylaxis. Curr Allergy Asthma Rep, 6(1), 9-13. https://doi.org/10.1007/s11882-006$\underline{0003-\mathrm{x}}$

Pawankar, R., Canonica, G.W., Holgate, S.T., \& Lockey, R.F. (2011). White Book on Allergy 2011-2012, World Health Organization. https://www.worldallergy.org/UserFiles/file/WAO-White-Book-onAllergy web.pdf

Rai, M, \& Jugal, K. (2009). Myths about diabetes and its treatment in North Indian population. International Journal of Diabetes in Developing Countries, 29(3), 129-132. https:/ / dx.doi.org/10.4103\%2F0973-3930.54290

Simmons, D. (2001). "Personal barriers to diabetes care: is it me, them, or us?: preface." Diabetes Spectrum 14(1), 1012. https://doi.org/10.2337/diaspect.14.1.10

Van de Velde, S., De Buck, E., Vandekerckhove, P., \& Volmink J. (2011). Evidence-Based African First Aid Guidelines and Training Materials. PLoS Med 8(7): e1001059. https://doi.org/10.1371/journal.pmed.1001059.

WHO (2016.) World Diabetes Report. https://www.who.int/diabetes/global-report/fr/ 